NASA Technical Memorandum 103123

AIAA-90-1899

\title{
Analysis of Internal Flow in a Ventral Nozzle for STOVL Aircraft
}

C. Frederic Smith

Sverdrup Technology, Inc.

Lewis Research Center Group

Brook Park, Ohio

and

Jack G. McArdle

National Aeronautics and Space Administration

Lewis Research Center

Cleveland, Ohio

Prepared for the

26th Joint Propulsion Conference

cosponsored by the AIAA, SAE, ASME, and ASEE

Orlando, Florida, July 16-18, 1990 


\title{
ANALYSIS OF INTERNAL FLOW IN A VENTRAL NOZZLE FOR STOVL AIRCRAFT
}

\author{
C. Frederic Smith* \\ Sverdrup Technology, Inc. \\ Lewis Research Center Group \\ Brook Park, Ohio 44142 \\ and \\ Jack G. McArdle $\dagger$ \\ National Aeronautics and Space Administration \\ Lewis Research Center \\ Cleveland, Ohio 44135
}

\section{Summary}

Short takeoff and vertical landing (STOVL) aircraft are planned for possible future development. For these aircraft, the same propulsion system will provide power for lift, hover, and horizontal flight. To accomplish this, many designs include a ventral nozzle to provide part of the vertical thrust required. Understanding and predicting the internal aerodynamic flow caused by a single exhaust duct opening are highly desirable in assessing this concept. This paper presents a numerical simulation of a ventral nozzle and compares the results with experimental data. Comparisons include visualizations of the flow along the ventral duct walls and in the tailpipe plane of symmetry. Performance calculations are also compared with measured values.

\section{Introduction}

Short takeoff and vertical landing (STOVL) aircraft are planned for possible future development. For these aircraft, the same propulsion system will provide power for lift, hover, and supersonic horizontal flight. In the lift mode the rear jet nozzle is blocked, and valves are opened to duct engine exhaust gases to two or more thrusters that are directed downward. In many proposed STOVL configurations, one of the lift

\footnotetext{
*Assistant Supervisor, Propulsion Aerodynamics Section. †Aerospace Research Engineer.
}

thrusters is a ventral nozzle which draws mixed core and fan gases from the engine tailpipe through a valve and opening, and has no inlet turning vanes. Close coupling between the tailpipe and the ventral nozzle is necessary because the valve and nozzle must be mounted wholly within the fuselage. The ventral nozzle also may swivel to provide trim and pitch control. An example of a general configuration of a ventral nozzle in an aircraft is shown in figure 1.

The concept of a ventral nozzle was explored in the early 1970's. The advantage of this approach for vertical thrust generation is that the nozzle can be positioned such that the total lift vector is located very near the aircraft's center of gravity. Early work on STOVL deflectors is reported by Wynosky et al. (refs. 1 and 2), whose experiments examined the internal flow field and performance of a turbofan engine and ventral nozzle test model configuration. A major concern discussed in reference 1 is the upstream static-pressure gradients created at the turbine exit by turning the flow $90^{\circ}$ into the ventral duct. These gradients, or flow distortions, could induce flow stability problems in the fan or turbine. The experiments indicate that this distortion diminishes rapidly with increasing distance from the ventral nozzle opening.

The objective of this current effort is to demonstrate the capability to numerically model the internal flow in a generic tailpipe and ventral nozzle configuration. To accomplish this goal, numerical predictions were made by solving the full threedimensional Navier-Stokes equations with an algebraic turbulence model. These results were compared with experimental data for one configuration. The comparisons include 
paint flow visualizations, total pressure surveys, wall static pressures, and performance data.

\section{Experiment Description}

A generic model tailpipe (about one-third full scale) with a single, large ventral nozzle was built and tested at NASA Lewis Research Center's Powered Lift Facility (PLF) to study the internal flow in this type of configuration. The facility was supplied with unheated air. A photograph of the experimental model is shown in figure 2. (Note that the model was tested upside down to simplify the experiment.) This model is based on a General Electric preliminary design proposed for the General Dynamics E-7D aircraft (ref. 3). The geometry of the experiment, although similar to that of the E-7D, was simplified to accommodate a straightforward grid-generation procedure for a numerical model of the flow field. However, the simplified experimental model still contains the essential flow physics of the E-7D.

A schematic of the experimental model is shown in figure 3 . As flow entered the tailpipe, it passed through a screen and a wire tripped the boundary layer. Then the flow was forced to turn $90^{\circ}$ to exit through the ventral nozzle. A blind flange at the end of the tailpipe simulated a blocked exhaust nozzle for the vertical flight mode. Measurements included flow, forces, and internal pressures. In addition, a thin plate was mounted along the model tailpipe centerline at the ventral opening to visualize the flow with dabs of oily paint. The paint ran along streamlines when airflow was established, and the resulting streaks provided a picture of the flow paths into the ventral opening. Paint flow visualizations were also performed along the ventral duct walls without the tailpipe flow visualization plate present. A detailed pitot-pressure survey was conducted at the nozzle exit (station 6b, fig. 3). Static pressures were also measured along the tailpipe and ventral duct/nozzle walls. For the data in this report, the ratio of tailpipe total pressure to ambient pressure was 2.96 .

\section{Numerical Analysis}

\section{PARC3D Code}

The analytical performance of this configuration was studied with the PARC3D code and the results were compared with experimental data to establish confidence in the ability of this code to reasonably predict the flow field in a tailpipe and ventral nozzle configuration. The PARC3D code was developed at the NASA Ames Research Center (ref. 4) for analyzing external flows. This code solves the compressible, threedimensional Navier-Stokes equations in generalized curvilinear coordinates with an algebraic turbulence model. It was later extended to analyze internal flows at the Arnold Engineering Development Center (ref. 5). PARC3D uses a Baldwin-Lomax turbulence model (ref. 6), and it solves the equations of motion by using the Beam-Warming approximate factorization scheme. Pulliam's scalar pentadiagonal transformation is applied to uncouple the equations of motion. This approach provides an efficient steady-state solver.

A block version of the PARC3D code was used. One advantage of this approach is that the computational grid is divided into two or more grid blocks to simplify the modeling of a complex geometry. Trilinear interpolation (ref. 7) is used to transfer information at grid block interfaces. Another advantage of using a block solver is that the majority of the information that needs to be stored in core memory is limited to the current block being analyzed. Therefore a problem involving a very large number of grid points may be solved with a computer that has less core memory than would be required to solve the problem by using two or more grid blocks.

\section{Grid and Boundary Conditions}

A "wire mesh" diagram of the tailpipe and ventral nozzle configuration is shown in figure 4. Since the experimental hardware had a geometric plane of symmetry, only half of the configuration was modeled for the computations. Since the tailpipe and ventral nozzle were at right angles to each other, it would have been very difficult to use one contiguous grid to model these components adequately. Therefore, the grid model was divided into two blocks: the tailpipe and the ventral nozzle. The tailpipe was modeled with an O-grid, and the ventral duct/nozzle was modeled with an $\mathrm{H}$-grid. The O-grid consisted of concentric circles that were parallel to the tailpipe surface and radial lines that were perpendicular to this surface. The $\mathrm{H}$-grid provided lines that were perpendicular and parallel to the ventral duct/nozzle walls. This approach provided body-conforming grids for each block.

These grids were generated algebraically by the INGRID3D code (ref. 8). The grids were stretched by hyperbolic stretching functions and were packed near the walls and near the center of the O-grid. Two grids were used for this study to evaluate the effects of the grid size on the predicted flow field. The first grid contained 31875 grid points in each block (51 by 25 by 25 points in the streamwise and orthogonal directions, respectively). This grid was limited by computer disk space available on the NASA Lewis Cray X-MP computer, but it was deemed adequate to evaluate the applicability of the PARC3D code to this problem. In order to provide an adequate number of points in the core flow region of the nozzle, grid packing near the nozzle surface was limited and the first grid point for the ventral nozzle was placed 0.1 in. from the surface. Three views of this grid are shown in figure 5. The results obtained with this grid were reasonable in comparison with the data.

A finer grid was generated for the NAS Cray Y-MP computer to explore the effects of grid density on the numerical solution. The finer grid contained 262701 points per block (101 by 51 by 51 points). Three views similar to the coarse grid are shown in figure 6 for comparison with figure 5. For 
the ventral nozzle, the first grid point was $0.01 \mathrm{in}$. from the surface. For the tailpipe in both grids, the first grid point was 0.01 in. from the surface. Comparisons of figures 5 and 6 indicate much denser packing of the grid near the walls and tailpipe center when the fine grid was used. The grid was packed near the center to minimize the effects of the "pole" boundary condition, which is discussed later.

The boundary conditions for this problem are shown in figure 7. Because it was desirable to keep the size of the computational grid as small as possible, the flow field outside of the nozzle was not analyzed. Although proper modeling of the nozzle exit flow conditions is very important, the application of boundary conditions at the nozzle throat is difficult because of the transonic nature of the flow. This problem was solved by adding a fictitious diverging section to the ventral nozzle to provide supersonic flow at the exit of the computational domain. This added section allowed the flow properties to be extrapolated downstream of the area of interest and modeled the nozzle throat as if the external plume were included. Previous studies have shown this to be a good approach to modeling a three-dimensional converging nozzle.

Another problem occurred at the center of the tailpipe O-grid: the radial grid lines became coincident at the center, causing singularity problems in the calculation of the coordinate transformation derivatives (metrics). To circumvent this problem, a so-called pole boundary condition was employed at the center of the O-grid. The flow properties were averaged around the adjacent grid line, and these average values were applied to all points along the innermost grid line, which has a radius of approximately 1 percent of the tailpipe radius.

\section{Results and Discussion}

The discussion in this section is divided into several topics. The comparisons of predicted particle trajectories with paint flow visualizations are discussed first. This is followed by comparisons of measured and predicted total and static pressures, along with performance parameters. The last topic concerns numerical modeling and computational aspects of the solutions obtained. The program PLOT3D (ref. 9) was used to generate the graphical presentation of the numerical results.

\section{Flow Visualization}

The following discussion compares paint flow visualizations and predicted particle trajectories obtained with the coarse and fine grids. These trajectories represent flow paths that massless particles would follow if they were released into the flow field. In figure 8, the tailpipe plane of symmetry paint flow results are compared with the fine and coarse grid solutions. As can be seen in the paint flows, a large vortex exists in the blocked tailpipe region. The predictions for the fine grid solution indicate several smaller vortices that are not present in the coarse grid solution. These small vortices are not apparent in the paint flow studies. This may be due to the paint not being able to flow within such small regions. The predicted vortex center is farther from the tailpipe centerline than the paint flows indicate. One source for this discrepancy may be the no-slip surface on the plate used in the visualization. The computed particle trajectories are along an inviscid plane of symmetry. The discontinuities in the particle paths at the tailpipe centerline resulted because the PLOT3D program did not recognize the pole at the center of the O-grid; however, the PARC3D solution is continuous across the centerline.

The upstream ventral duct wall paint flows are shown in figure 9 along with the predicted particle trajectories. (The nomenclature for the ventral duct walls is presented in fig. 6.) The two solutions are similar to each other and compare well with the experimental data. As can be seen from the flow patterns, the ventral duct vortex pulls fluid towards the plane of symmetry from the side wall of the duct. The two counterrotating vortices can be seen in the paint streaks and the particle trajectories along the duct wall moving towards each other.

The downstream ventral-duct wall flow visualization is shown in figure 10 along with predictions. In this region, the finer grid provides much better agreement with the experiment than the coarse grid does. In this figure, the flow is split into two distinct directions. Near the duct inlet, the flow tends to move back towards the duct inlet, which represents a region of separated flow. Farther along the duct wall, the flow moves away from the side ventral duct wall and towards the nozzle exit.

The side duct wall paint flows are shown in figure 11. Both accompanying predictions agree well qualitatively with the experimental data. In this figure the flow tends to move from the downstream wall towards the upstream duct wall. This movement was due to the presence of the nozzle vortices.

\section{Total and Static Pressures}

The pitot pressures measured in the nozzle exit plane and the total pressure field predicted by the PARC3D code are shown in figure 12. An oval-shaped region of low pressure is observed near the upstream nozzle wall in both the measured data and the numerical results. The solutions obtained with the coarse and fine grids provide similar results. The fine grid results indicate a stronger vortex than the coarse grid solution indicates. Measured and predicted flow-angle surveys show that the nozzle flow from the downstream and side walls is moving towards this region. The predicted contours of local total to upstream total pressure ratios greater than 1.01 are restricted to regions very close to the ventral duct walls. This is not apparent in the color contour plots shown.

These flow characteristics are exhibited well in the predicted particle trajectories shown in figure 13. A major feature shown in this figure is the large vortical region near the upstream wall of the ventral duct. The lower pressure in this region drew flow from the downstream and side ventral duct walls. The vortex persisted through the nozzle exit. This vortical region is shown in the paint flow visualization of 
the upstream ventral duct wall and in the total-pressure surveys at the nozzle exit. The low-pressure region caused the jet to exit the nozzle with an upstream-facing velocity component, thus producing a significant downstream component of thrust which was measured to be approximately 10 percent of the vertical thrust. The predicted downstream component, determined from the calculated average flow angles, is approximately 8 percent of the vertical thrust. As was shown in the experimental flow visualizations, there were counterrotating vortices present in the ventral duct. These vortices are consistent with the symmetric nature of the test hardware. The second vortex is assumed to be present in the calculations by the plane of symmetry boundary condition. The calculated tailpipe wall static pressures are within 2 percent of the measured data, and the predicted wall static pressures near the nozzle exit are within approximately 3 percent of the data.

Figure 14(a) shows how the flow turning into the ventral duct affects the static-pressure gradients in the tailpipe. The static-pressure field distortion decreases rapidly as the distance from the ventral nozzle opening increases. This trend implies that the ventral duct may not affect turbine or fan flow stability unless the turbine or fan is placed very close to the nozzle opening. This result is consistent with trends reported in references 1 and 2 . The static pressures in the ventral duct and nozzle are shown in figure 14(b). Large gradients in the static-pressure field due to the vortex present in the nozzle opening are observed. The flow field distortion is also apparent in the Mach number contours shown in the tailpipe and ventral nozzle shown in figure 15 . In figure 15 (a), a rapid acceleration of the flow around the upstream ventral duct corner can be seen. In figure 15(b), the presence of the vortex is indicated by the large gradients in the somewhat circular Mach number contours that are centered near the upstream plane of symmetry corner of the ventral duct and nozzle.

\section{Performance}

The predicted thrust and mass flow obtained from the coarse and fine grid solutions are compared with experimental data in table I. The \pm numbers indicate the amount of oscillation in the solution. As can be seen, the finer grid exhibited a reduced amplitude. These oscillations may be due to the inherent unsteadiness of flows containing large vortical regions. The thrust predictions are in good agreement with the measured thrust, and the predicted mass flow compares well with the experimental data.

\section{Modeling and Numerical Issues}

\section{Static Pressure}

The discrepancies between the measured and predicted static pressures may be due, in part, to the turbulence model used. The Baldwin-Lomax turbulence model was developed for two-
TABLE I.-VENTRAL NOZZLE PERFORMANCE

\begin{tabular}{|c|c|c|c|}
\hline \multirow[t]{2}{*}{ Performance indicators } & \multicolumn{2}{|c|}{$\begin{array}{l}\text { Numerical } \\
\text { results }\end{array}$} & \multirow[t]{2}{*}{$\begin{array}{l}\text { Experimental } \\
\text { results }\end{array}$} \\
\hline & Coarse grid & Fine grid & \\
\hline $\begin{array}{l}\text { Tailpipe to ambient } \\
\text { pressure ratio, }{ }^{\mathrm{a}} P_{5} / P_{\mathrm{amb}}\end{array}$ & 2.97 & 2.96 & 2.96 \\
\hline $\begin{array}{l}\text { Nozzle inlet to tailpipe } \\
\text { pressure ratio, }{ }^{\text {a }} P_{6} / P_{5}\end{array}$ & .932 & .935 & .945 \\
\hline $\begin{array}{l}\text { Nozzle discharge coefficient } \\
\text { based on }{ }^{\text {a }} P_{5}\end{array}$ & .88 & .91 & .91 \\
\hline $\begin{array}{l}\text { Nozzle thrust coefficient } \\
\text { based on }{ }^{\text {a }} P_{5}\end{array}$ & .96 & .97 & .97 \\
\hline Corrected flow rate, ${ }^{b} \mathrm{lbm} / \mathrm{sec}$ & $22.67 \pm 0.2$ & $23.32 \pm 0.04$ & 23.04 \\
\hline Corrected thrust, ${ }^{\mathrm{c}}$ lbf & $870 \pm 9$ & $905 \pm 2$ & 896 \\
\hline Corrected axial thrust, ${ }^{\mathrm{c}} \mathrm{lbf}$ & -88 & -72 & -73 \\
\hline
\end{tabular}

${ }^{\mathrm{a}}$ Where $P_{5}=$ total pressure at station 5. $P_{6}=$ total pressure at station 6 , and $P_{\text {amb }}=$ total ambient pressure

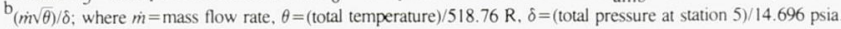
${ }^{\mathrm{c}} F / \delta$; where $F=$ thrust

dimensional, separated flows, and the extension of this model to three-dimensional flows is difficult, especially in cases where there are multiple walls (such as the ventral duct/nozzle). The turbulent viscosities calculated for each wall were weighted and averaged on the basis of the distance from the associated wall to the point of calculation. In addition, the ventral duct flow contained a large vortical region that may not be accounted for adequately in the model. A two-equation turbulence model, such as a k- $\epsilon$ model, would handle the multiple walls implicitly for the turbulence model equations.

\section{Boundary Layer Resolution}

Another consideration is that the boundary layer may not be resolved well enough. The $\mathrm{y}^{+}$distance for the first grid point off the ventral duct wall was typically 15 for the fine grid, where

$$
\mathrm{y}^{+}=\frac{\text { normal distance }(\text { shear stress/density) })^{0.5}}{\text { kinematic viscosity }}
$$

This distance is slightly outside of the viscous sublayer region of the boundary layer. For a fully defined boundary layer, the first grid point in the flow field should be located at a $\mathrm{y}^{+}$ distance of less than 10 from the surface (ref. 10). However, decreasing the $\mathrm{y}^{+}$for the first grid point off the wall from 150 (for the coarse grid) to 15 (for the fine grid) did not significantly change the results for the wall static-pressure calculations. This may be due to the fact that the separation was generated by the sharp corner at the ventral duct opening and not by adverse pressure gradients. 


\section{Conservation of Mass}

The predicted inlet and exit mass flow variations for the fine grid solution as a function of number of time steps are shown in figure 16 . The differences may be partially attributed to the fact that the transfer of flow properties at the interface between the grid blocks is not forced to be conservative or characteristically correct. As the solution is iterated, the amplitude of the oscillations and the discrepancies in the inlet and exit mass flow rates diminish. The residuals (differences in flow properties between successive iterations) were reduced approximately three orders of magnitude. Difficulties encountered in further reducing the size of the residuals may be attributable to the turbulence model or possible flow field unsteadiness.

\section{Low Mach Number Effects}

Another contributing factor to the convergence difficulties is the low Mach number flow in the blocked region of the tailpipe. Typical Mach numbers are less than 0.1 in this region; such low Mach levels are a classic source of numerical problems for compressible flow codes. Preconditioning could improve this situation (ref. 11).

\section{Computational Issues}

The maximum Courant-Friedrichs-Lewy (CFL) number (ref. 12) that could be used for both blocks to obtain a stable solution was 1.0 for the coarse grid and 0.5 for the fine grid. (The CFL number is the time step limiter). The computational speed for the coarse grid was 800 iterations per CPU hr on the Cray X-MP. The fine-grid speed was 200 iterations per $\mathrm{CPU}$ hr on the Cray Y-MP. The coarse-grid solution required approximately 4000 iterations, whereas the fine-grid solution required 12000 iterations.

\section{Conclusions and Recommendations}

To understand and predict the internal flow in a tailpipe and ventral nozzle configuration, a comparison was made between the numerical results obtained from the PARC $3 \mathrm{D}$ code and experimental data for this model.

The experimental and numerical results showed that the flow field within a tailpipe and ventral nozzle configuration is extremely complex. The results of this study demonstrate that the PARC3D code can predict realistic flow fields and performance for such a configuration. The numerical solutions are somewhat grid dependent. Several flow field details exhibited in the fine-grid results are not present in the coarse-grid solutions, although reasonable predictions of the major flow field phenomena and nozzle performance parameters were made using the coarse grid. The analysis reconfirmed previous experimental results that the flow turning into the ventral duct opening has a limited effect on the upstream flow distortion in the tailpipe for the present configuration.

One major step for improving the numerical model would be to apply a two-equation turbulence model in place of the algebraic model currently used. The two-equation model would handle multiple walls in a more straightforward manner. A $\mathrm{k}-\epsilon$ (two-equation) turbulence model is being incorporated into the PARC3D code. The computational time required to use such a turbulence model will increase considerably.

The flow field has large regions of very low speed flow which can impede convergence for compressible flow solvers. The inclusion of preconditioning may alleviate these problems.

\section{Acknowledgments}

This work was managed by Peter G. Batterton under NASA Contract NAS3-25266. Part of the analysis was performed utilizing the Numerical Aerodynamic Simulation (NAS) facility located at the NASA Ames Research Center.

\section{References}

1. Wynosky, T.A., and Szyszko, C.J., "V/STOL Deflector Aerodynamic Design Criteria-Geometric Variations Effects,' AIAA Paper 73-1181, Nov. 1973

2. Wynosky, T.A., Streib, R.A., and Usab, W.J., "V/STOL Deflector Concepts," AIAA Paper 74-1168, Oct. 1974.

3. Meyer, B.E., Re, R.J., and Romine, B.M., "'A Static Investigation of Several STOVL Exhaust System Concepts,'” AIAA Paper 89-2928, July 1989.

4. Pulliam, T.H., and Steger, J.L., "Implicit Finite-Difference Simulations of Three Dimensional Compressible Flow,' AIAA Journal, Vol. 18, No. 2, Feb. 1980, pp. 159-167.

5. Cooper, G.K., Jones, R.R., Phares, W.J., and Swafford, T.W., "Application of Computational Fluid Dynamics to Test Facility and Experiment Design,' AIAA Paper 86-1733, June 1986.

6. Baldwin, B.S., and Lomax, H., "Thin-Layer Approximation and Algebraic Turbulence Model for Separated Turbulent Flows,'” AIAA Paper 78-257, Jan. 1978.

7. Stokes, M.L., and Kneile, K.L., “'A Search/Interpolation Algorithm for CFD Analysis,' Presented at the World Congress on Computational Mechanics, University of Texas, Austin, TX, Sept. 1986.

8. Soni, B.K., "Two- and Three-Dimensional Grid Generation for Internal Flow Applications of Computational Fluid Dynamics," AIAA Paper 85-1526, July 1985.

9. Wlatka, P.P., and Buning, P.G., "PLOT3D User's Manual,', NASA TM-101067, 1990.

10. Kuethe, A.M., and Chow, C.Y.: Foundations of Aerodynamics, Bases of Aerodynamic Design, 4th ed., John Wiley and Sons, Inc., New York, 1986.

11. Choi, Y.H., "Computation of Low Mach Number Compressible Flow," Ph.D. Thesis, The Pennsylvania State University, 1989.

12. Courant, R., Friedrichs, K.O., and Lewy, H., "Uber die Partiellen Differenzengleichungen der Mathematischen Physik," Mathematische Annalen, Vol. 100, 1928, pp. 32-74. (Translated to: “'On the Partial Difference Equations of Mathematical Physics,' IBM Journal of Research and Development, Vol. II, 1967, pp. 215-243. 


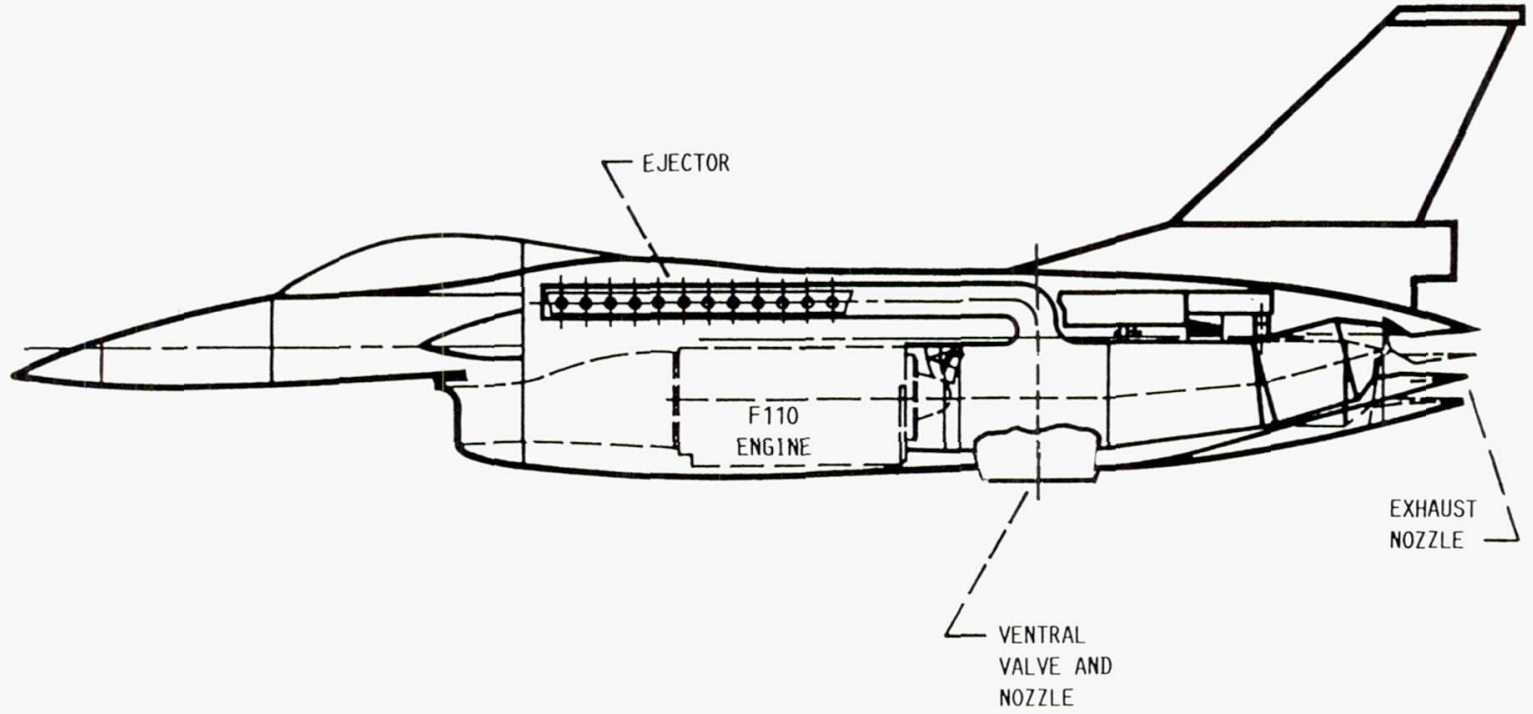

Figure 1.-Ventral nozzle concept.

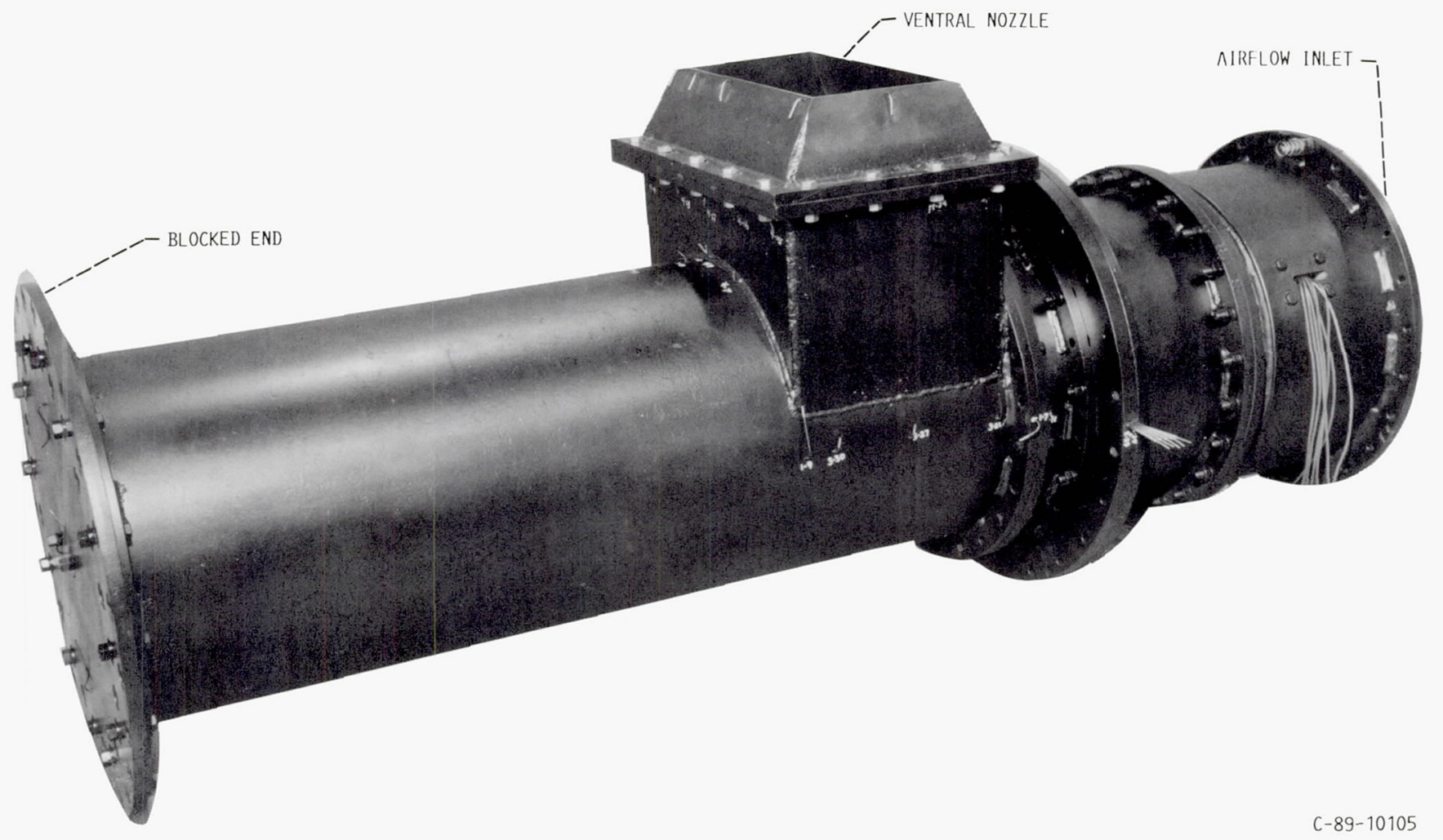

Figure 2.-Tailpipe and ventral nozzle experimental hardware. (Note that the model was tested upside down to simplify the experiment.) 


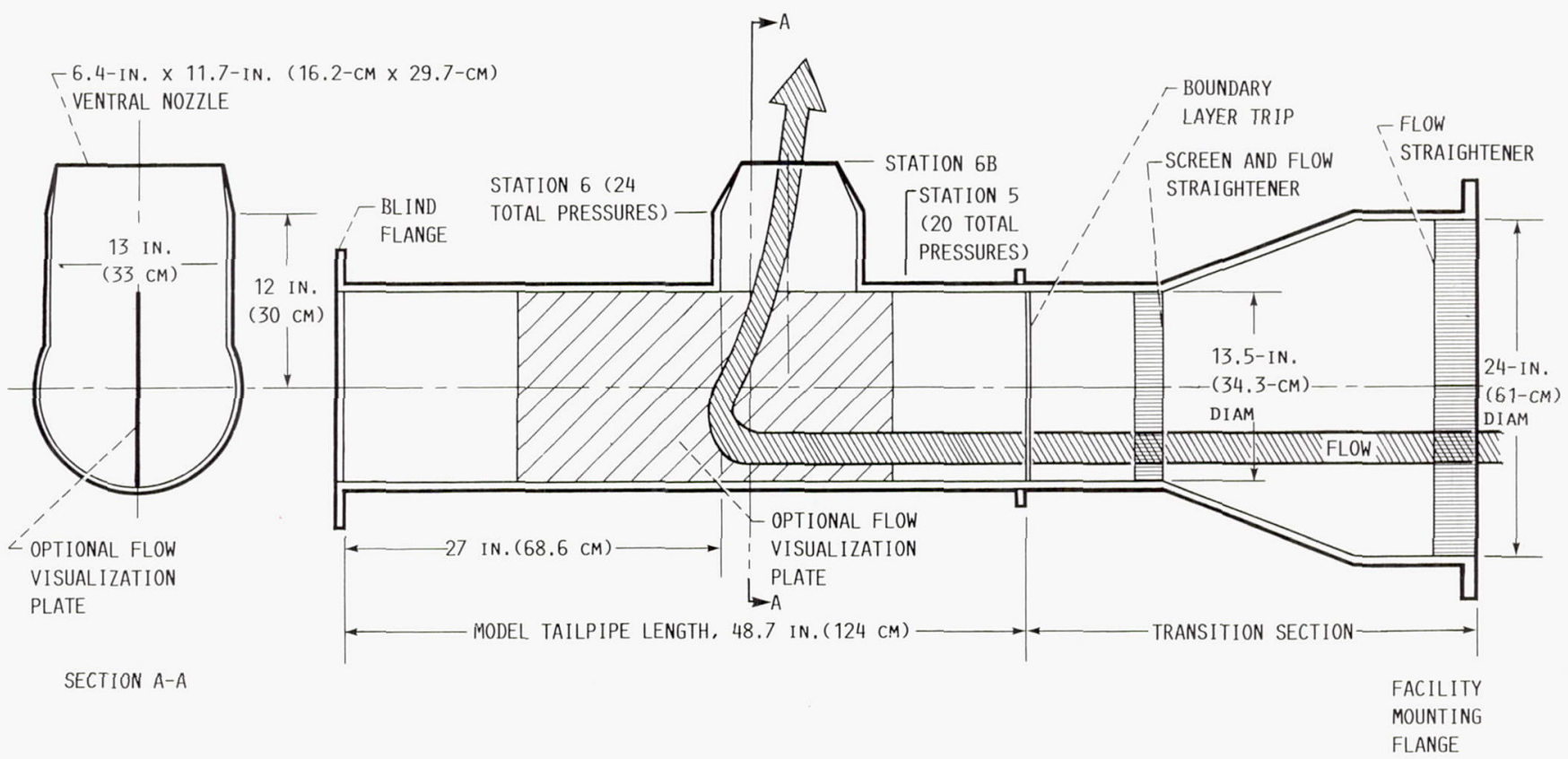

Figure 3.-Schematic of experimental model.

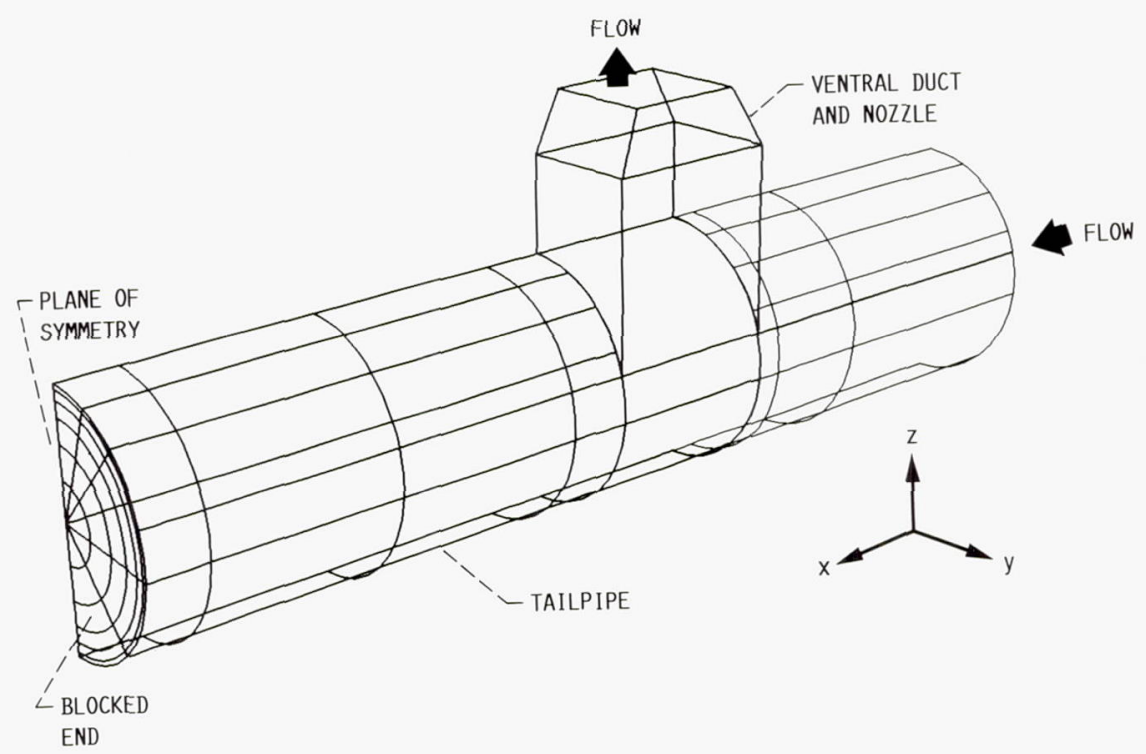

Figure 4.-"Wire mesh" model of tailpipe and ventral nozzle. 


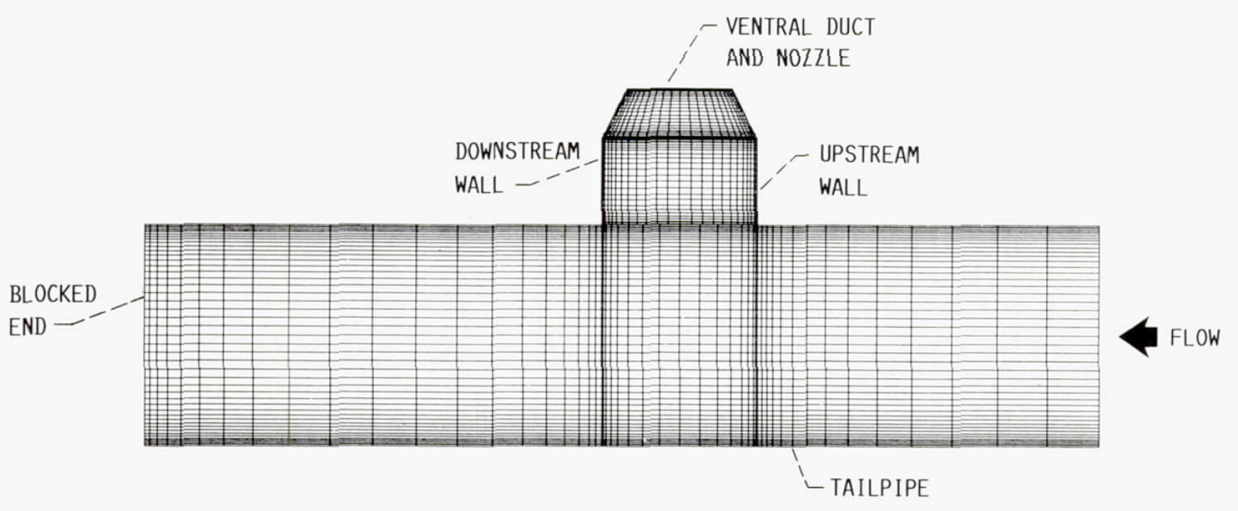

(a)

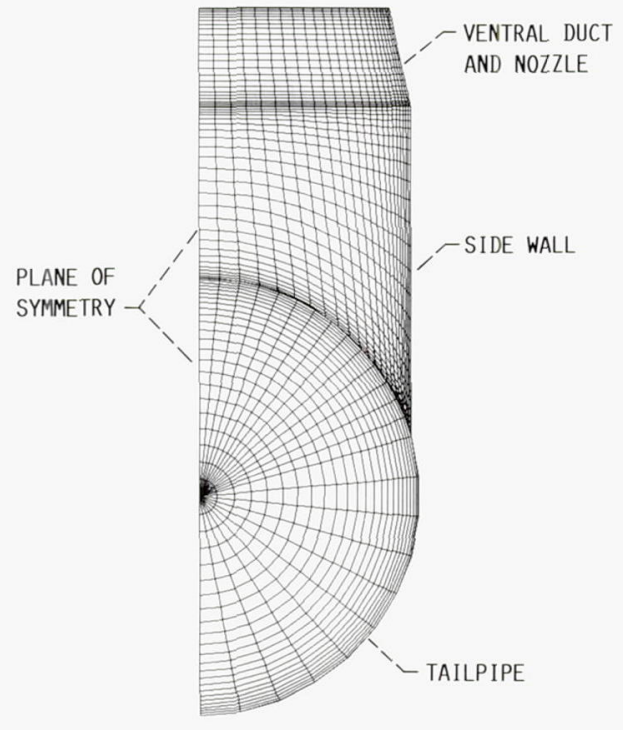

(b)

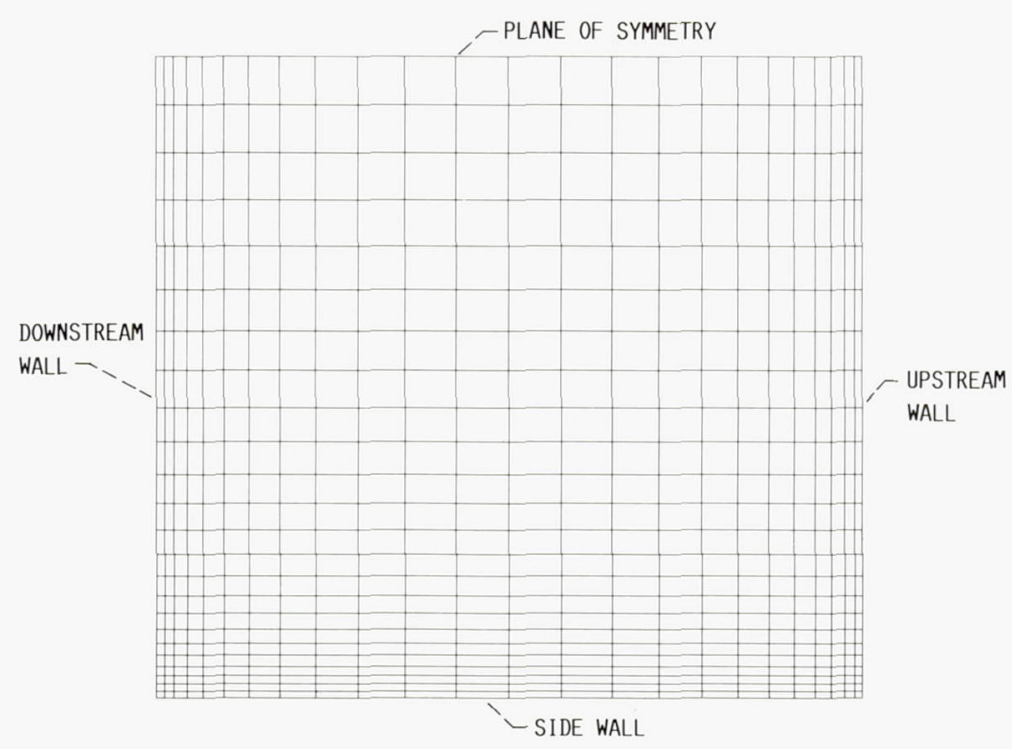

(c)

(a) Plane of symmetry (xz-plane).

(b) End view (yz-plane).

(c) Nozzle exit (xy-plane).

Figure 5.-Coarse grid. 


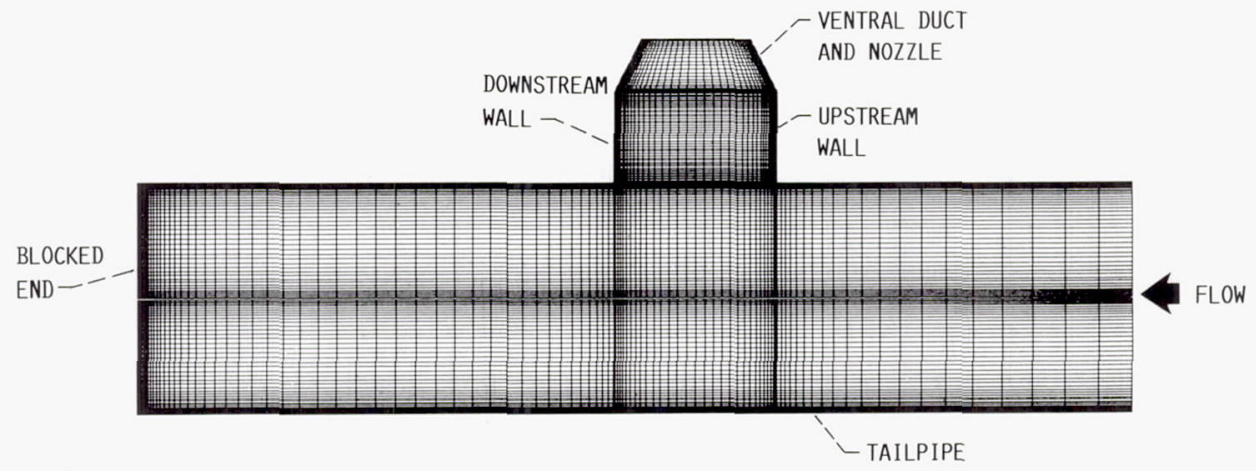

(a)

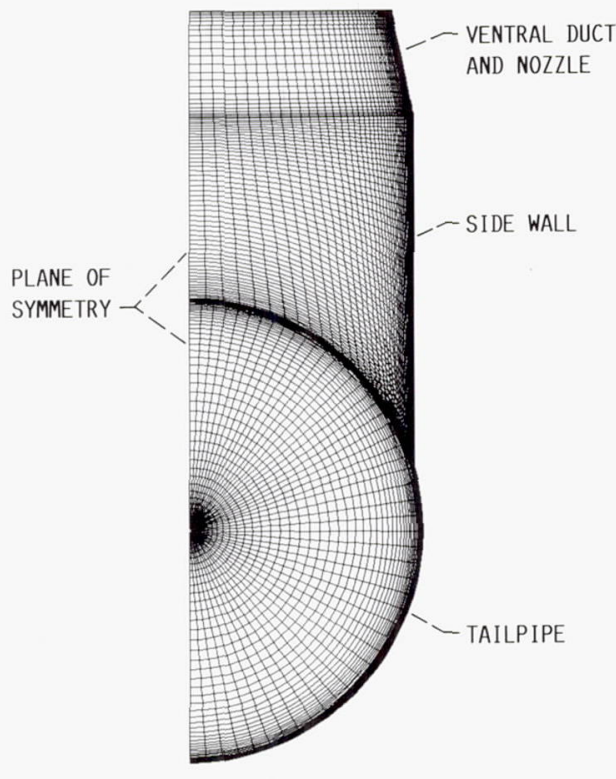

(b)

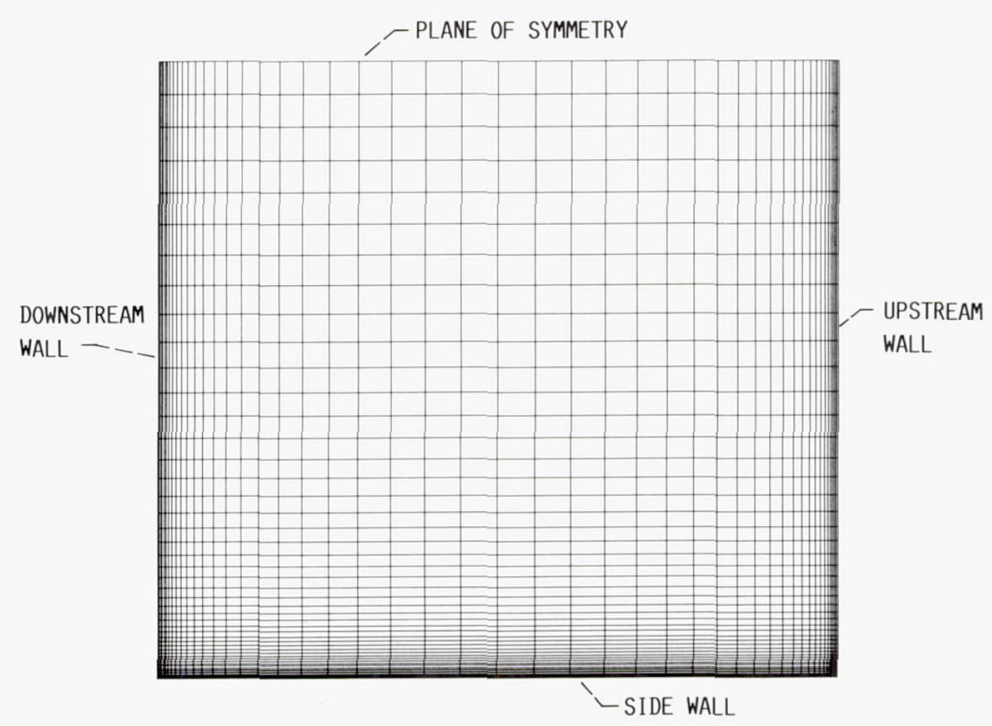

(c)

(a) Plane of symmetry (xz-plane)

(b) End view (yz-plane).

(c) Nozzle exit (xy-plane).

Figure 6.-Fine grid. 


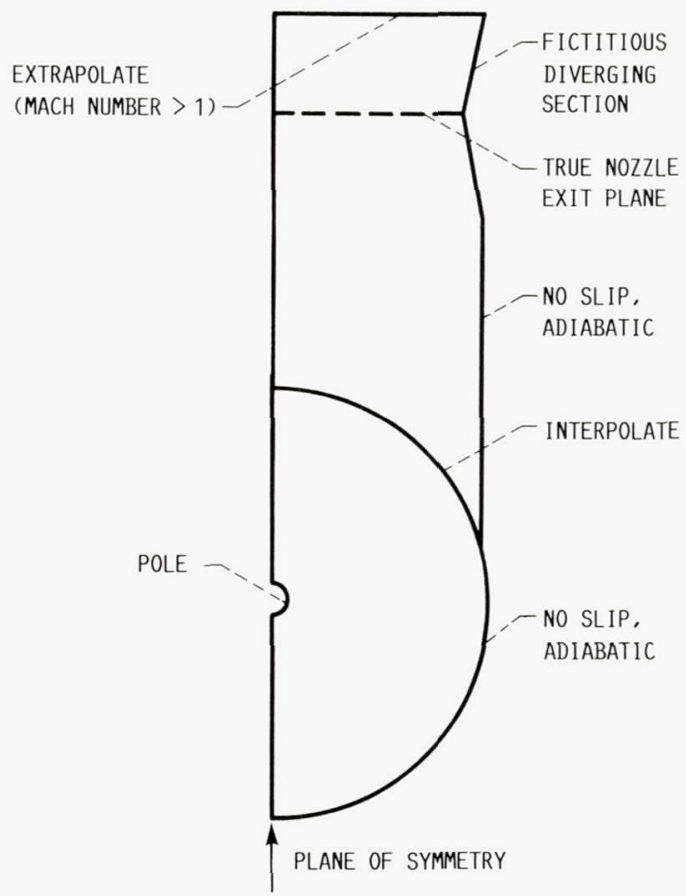

Figure 7.-Boundary conditions. Total pressure and total temperature were specified for upstream portion of tailpipe; adiabatic conditions and no slip were specified for downstream portion of tailpipe. 

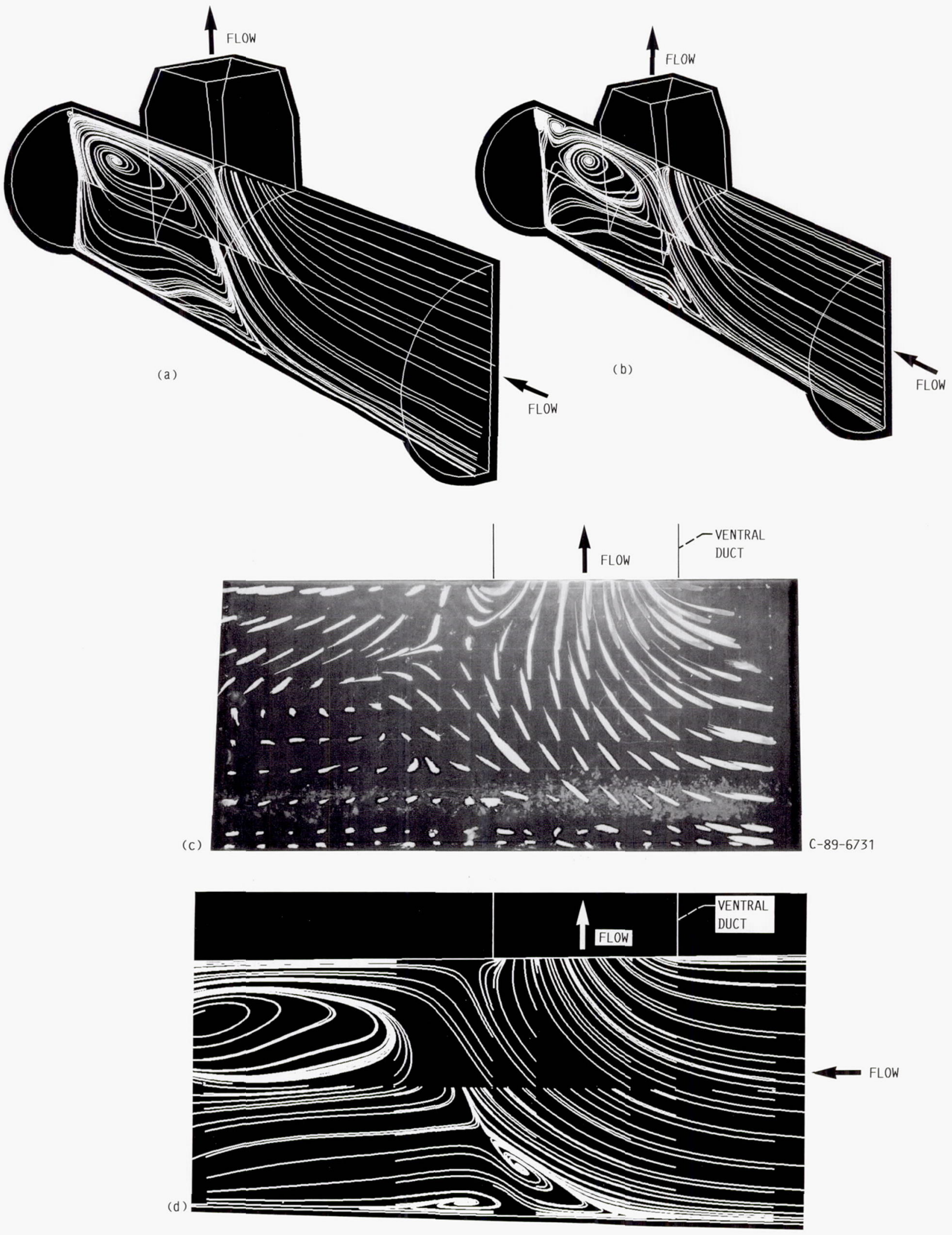

$\begin{array}{ll}\text { (a) Coarse grid. } & \text { (b) Fine grid }\end{array}$

(c) Experiment.

(d) Prediction

Figure 8.-Tailpipe plane of symmetry flow visualization. 

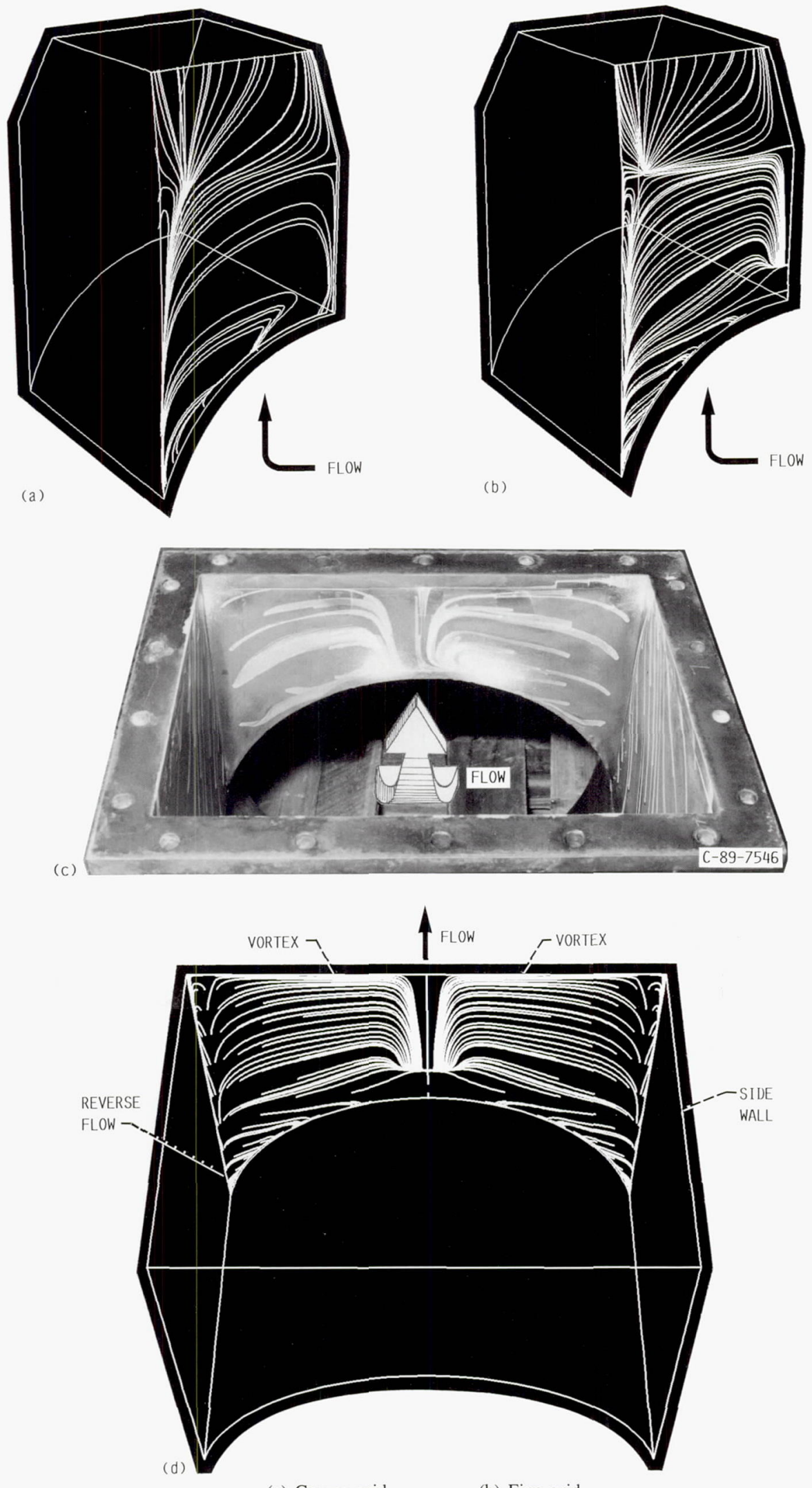

(a) Coarse grid.

(b) Fine grid.

(c) Experiment. (Nozzle not present.)

(d) Prediction. (Nozzle not present; solution reflected about plane of symmetry.)

Figure 9.-Upstream ventral duct and nozzle wall flow visualization. 

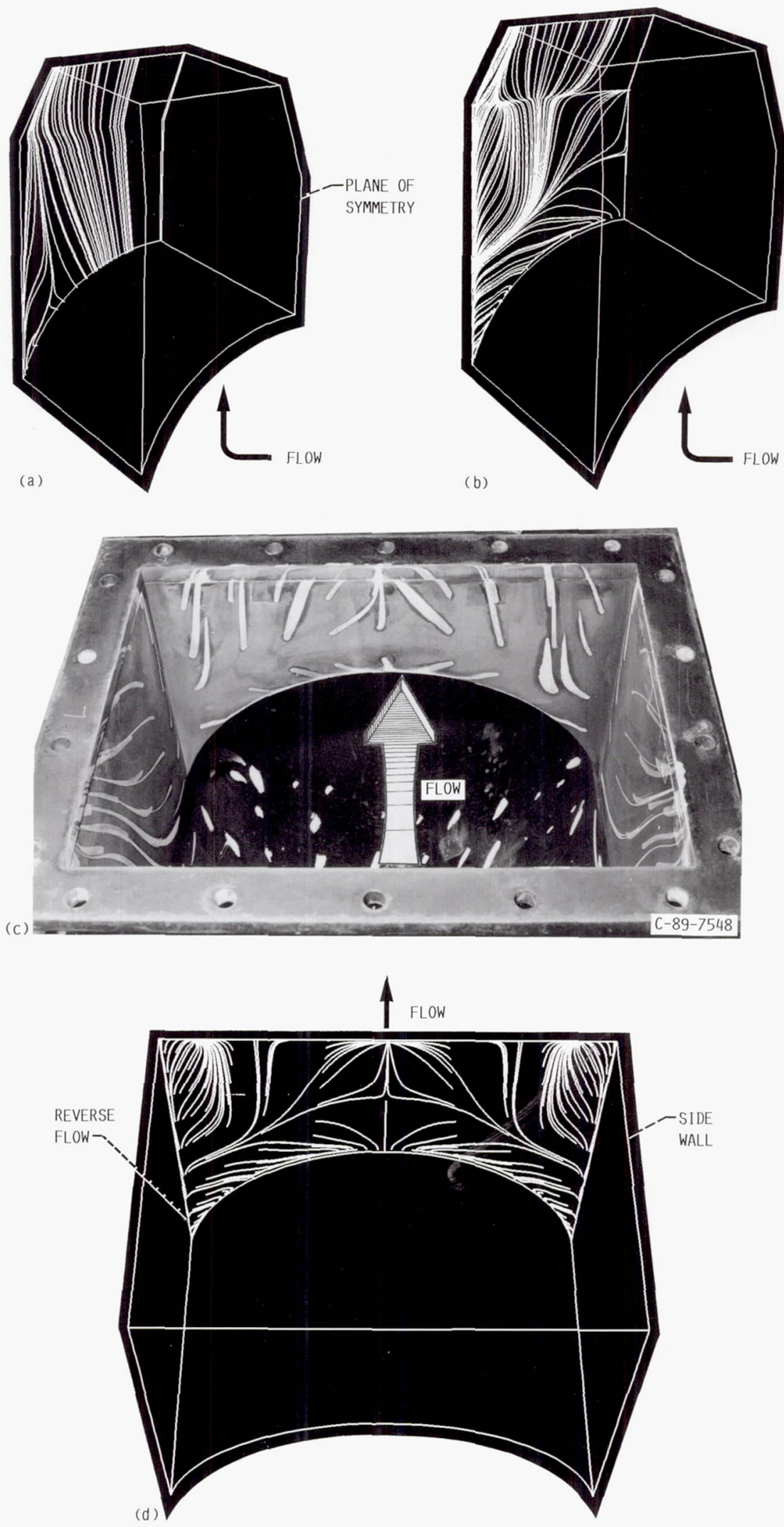

$\begin{array}{ll}\text { (a) Coarse grid. } & \text { (b) Fine grid. }\end{array}$

(c) Experiment. (Nozzle not present.)

(d) Prediction. (Nozzle not present; solution reflected about plane of symmetry.)

Figure 10.-Downstream ventral duct and nozzle wall flow visualization. 

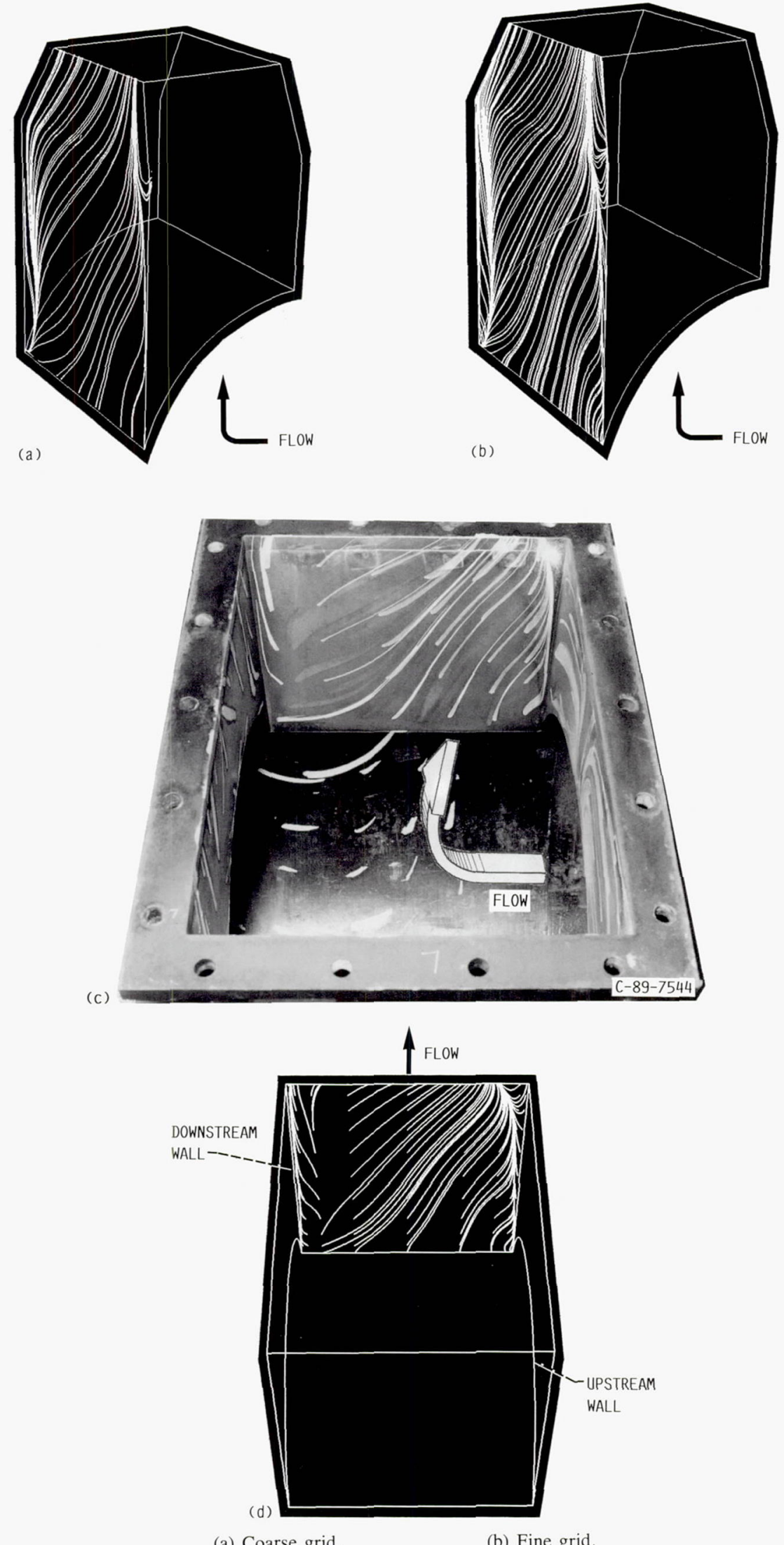

(a) Coarse grid.

(c) Experiment. (Nozzle not present.)

(d) Prediction. (Nozzle not present.)

Figure 11.-Side ventral duct and nozzle wall flow visualization. 


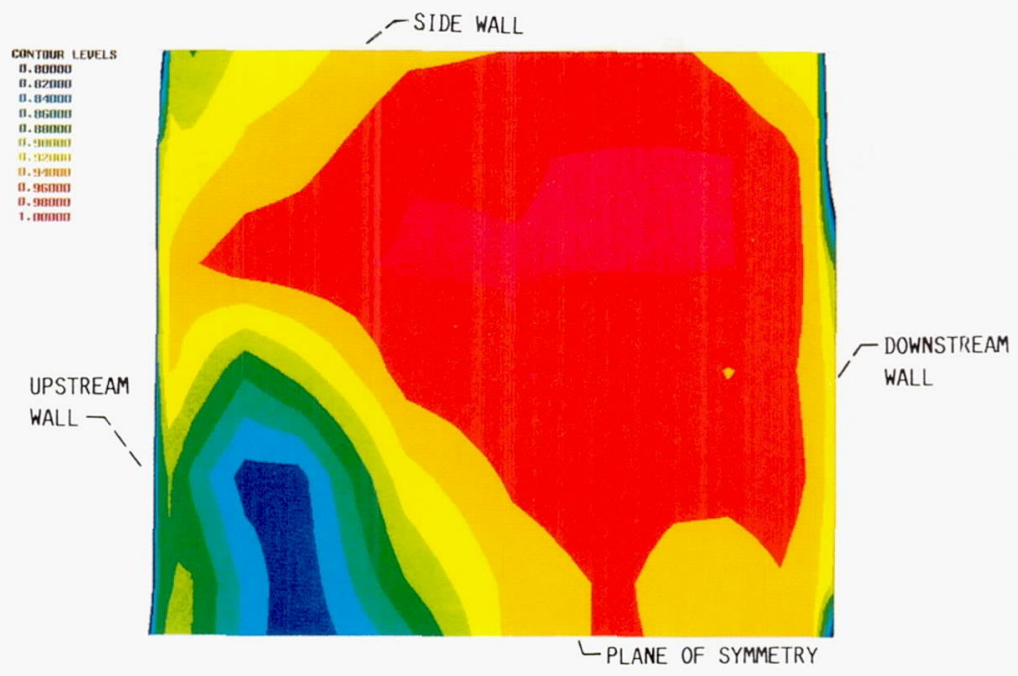

(a)

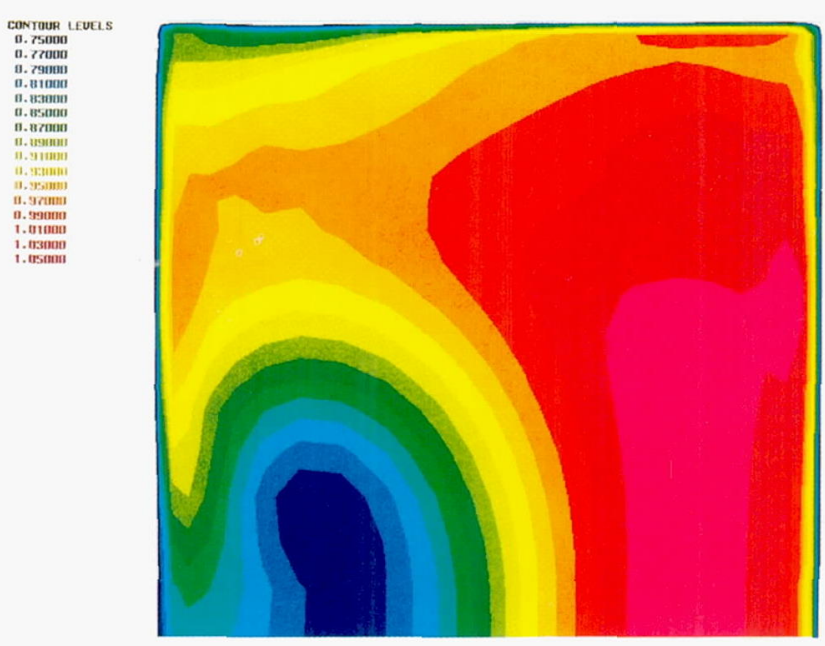

(b)

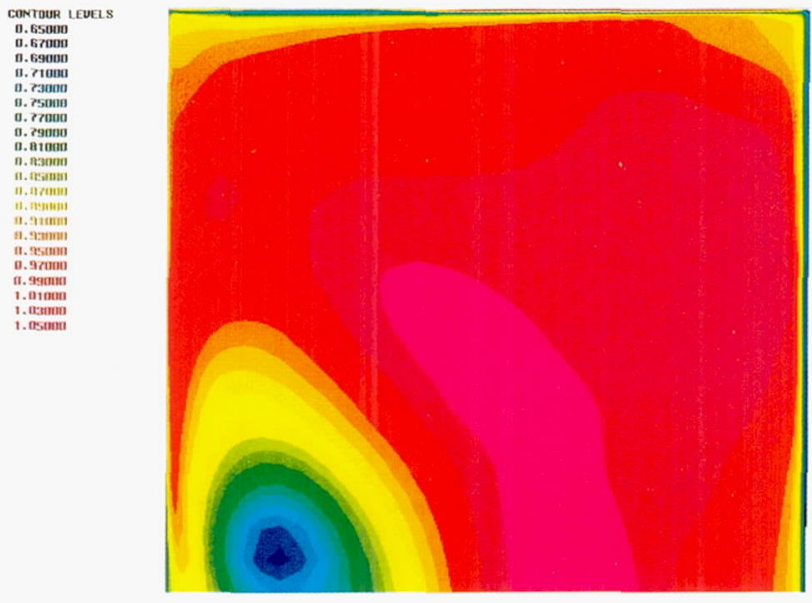

(c)

(a) Measured pitot pressure.

(b) Coarse grid total pressure.

(c) Fine grid total pressure.

Figure 12.-Nozzle exit plane pitot and total pressures. (The contours represent levels of local total to upstream total pressure ratios.) 


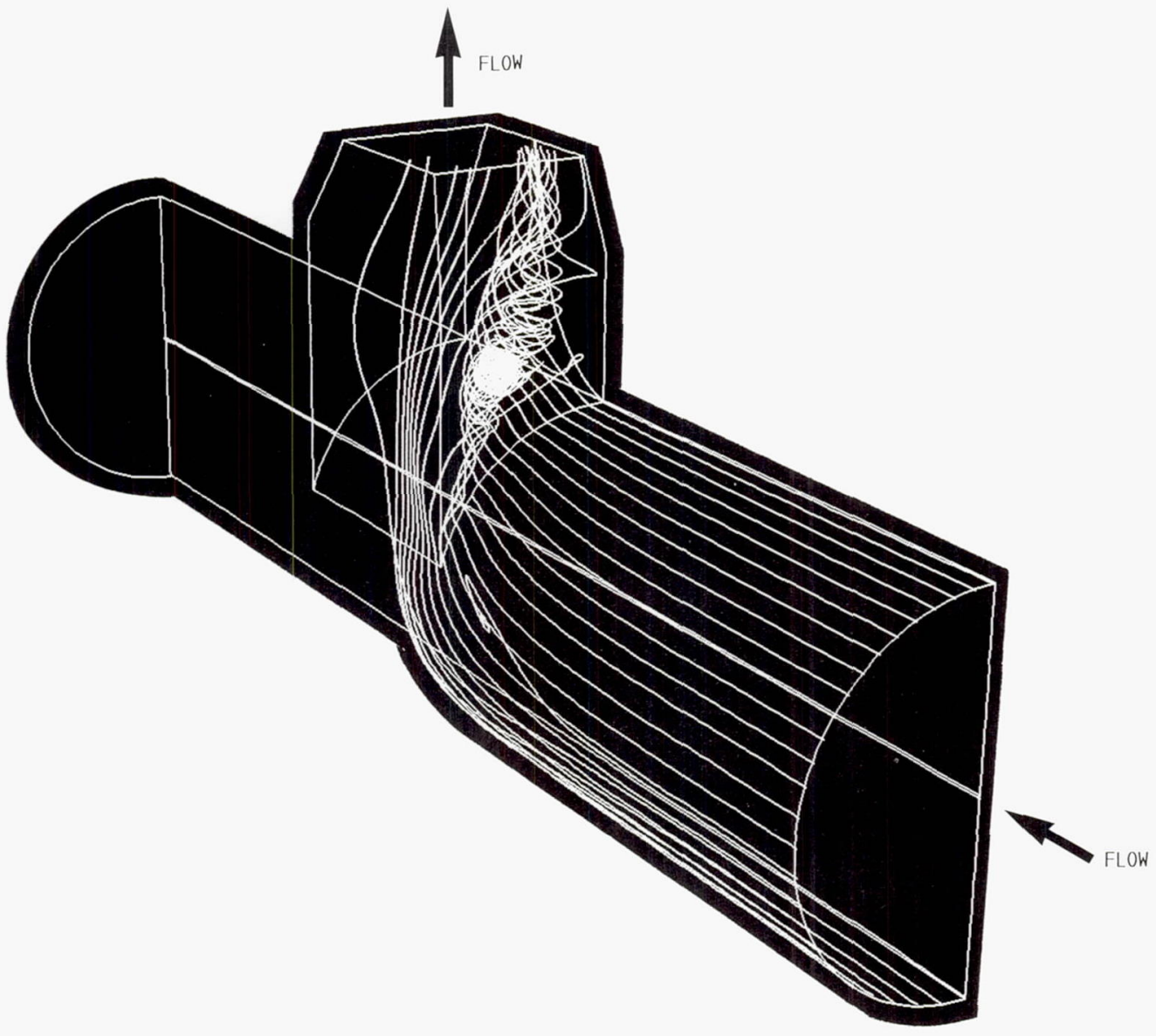

Figure 13.-Predicted particle trajectory paths 


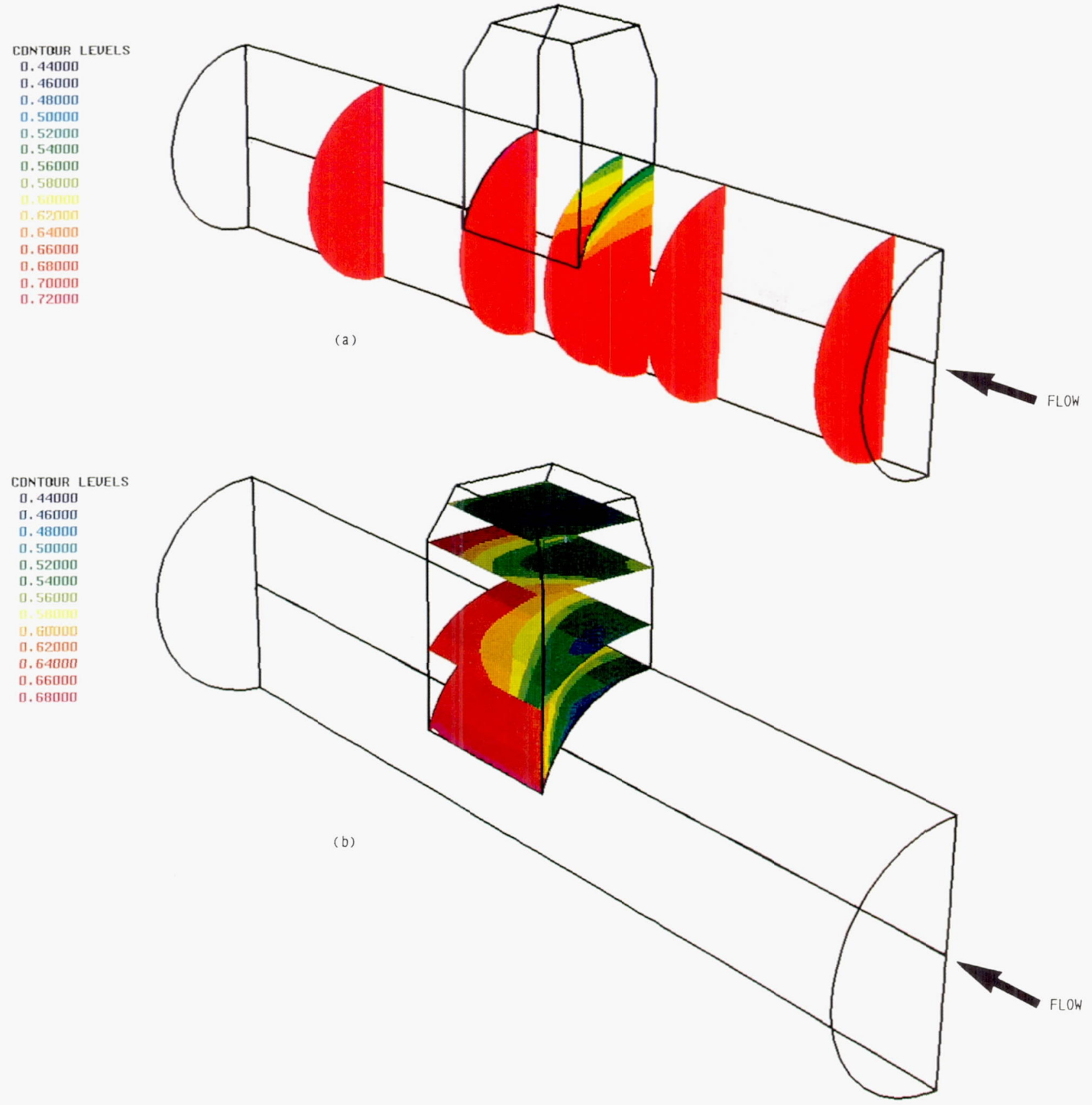

(a) Tailpipe.

(b) Ventral duct and nozzle.

Figure 14.-Predicted static pressure. (Contours represent levels of normalized local static pressure.) 
0. 15000

0.20000

0. 25000

0. 30000

0.35000

0.457101

0.50000

0. 55000

0.60000

0. 65000

0. 20000

CDNTOUR LEUELS

0.05000

0.15000

0.20000

0.25000

0.30000
0.35000

0. 55000

0. 60000

0. 65000

0.70000

0.75000
0.80000

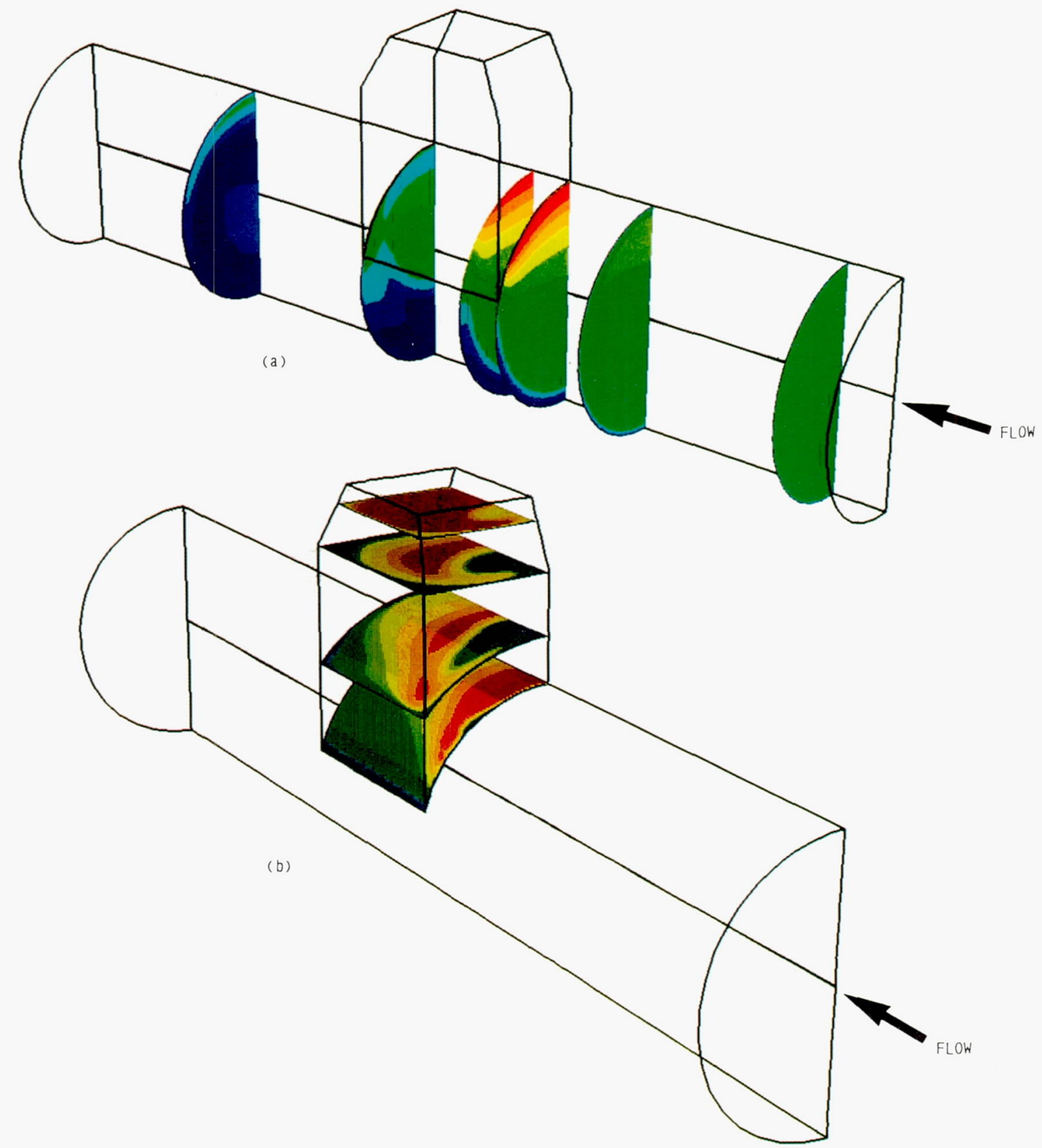

(a) Tailpipe

(b) Ventral duct and nozzle.

Figure 15.-Predicted Mach number. 


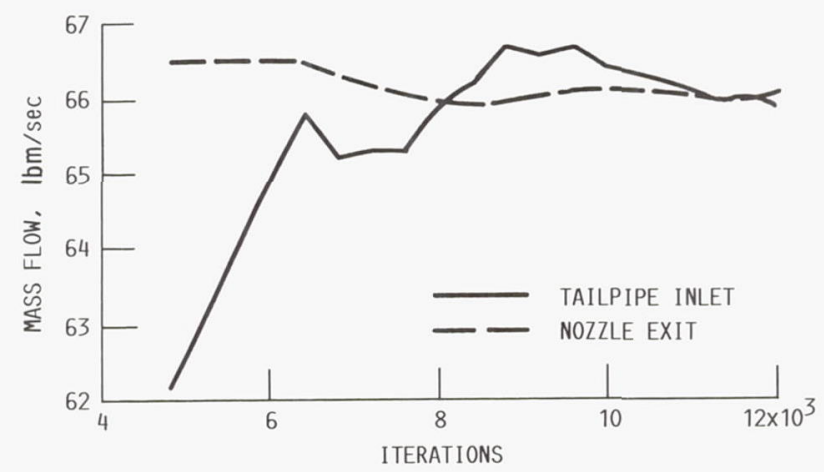

Figure 16.-Calculated mass flow rates as function of number of time steps. 


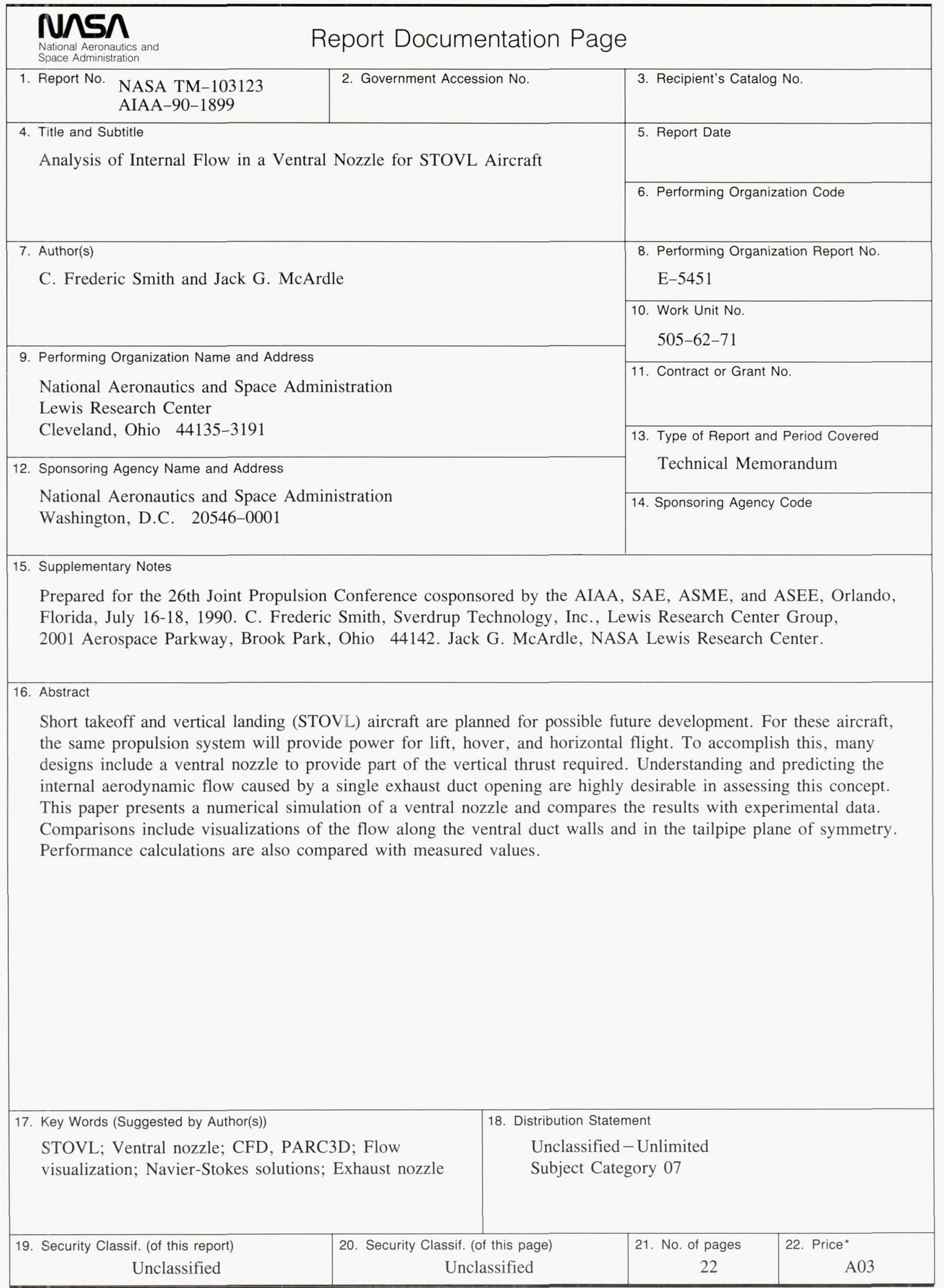

